\title{
Medizinstudium über das Internet
}

R. Ster, A. Pril

Korrespondenz:

Prof. Dr. med. Ruedi Ster Fachbereich Medizin der Fernuniversität Frauenfeld Schaffhauserstrasse 340 a CH-3500 Frauenfeld

ruedi.ster[at]gmx.ch
Ab 1940 stieg die Zahl der berufstätigen Ärztinnen und Ärzte in der Schweiz exponentiell an. Immer mehr Medizinstudenten drängten in die überquellenden Hörsäle. Doch in den 1990er Jahren sorgte das Damoklesschwert einer medizinischen Überversorgung für einen Strategiewechsel: mit der Einführung des Numerus clausus bzw. Eliminationsprüfungen nach dem ersten Studienjahr, der Verdoppelung der Regelstudienzeit von drei auf sechs Jahre und Vorlesungen über medizinische Statistik wurde gezielte Abschreckungspropaganda gegen die Aufnahme des Medizinstudiums betrieben - und die Studentenzahlen in den Folgejahren drastisch gesenkt.

Seit geraumer Zeit ist jedoch nicht mehr von einer Ärzteschwemme, sondern von einem bedrohlichen Ärztemangel die Rede. Während pro Jahr 600 bis 800 junge Mediziner/innen ihre Ausbildung abschliessen, gehen mehr als 1000 ältere in Pension. Bereits heute sieht sich die Schweiz nicht mehr in der Lage, ihren Bedarf aus eigener Kraft zu decken. Fast die Hälfte der Ärztinnen und Ärzte in Schweizer Spitälern kommt aus dem Ausland, die Mehrzahl von ihnen aus Deutschland [1]. Verfügen Patienten und das Schweizer Personal jedoch nicht über ausreichende Kenntnisse des Schriftdeutschen, gibt es Kommunikationsschwierigkeiten - und die Bewältigung des Klinikalltags wird selbst in der Deutschschweiz zu einem Problem [2].

Noch prekärer stellt sich die Situation bei den niedergelassenen Kollegen dar, insbesondere in den ländlichen Regionen: jeder zweite praktizierende Allgemeinmediziner ist über 55 Jahre alt und wird in absehbarer Zeit seine Tätigkeit einstellen, während es die jüngeren Kolleginnen und Kollegen in die Grossstadt zieht. Die Autoren einer aktuel-

len Studie schätzen, dass es in 30

Jahren nicht einmal mehr 400 niedergelassene Ärztinnen und Ärzte ausserhalb der Agglomerationen geben wird und dass bei einem geschätzten Frauenanteil von 93\% die Praxen im Durchschnitt nur noch zwei Tage pro Woche geöffnet sein werden sofern wirksame politische Gegenmassnahmen ausbleiben [3].

Neben der gezielten Förderung der Hausarzt- medizin drängt sich förmlich die Schaffung zusätzlicher Studienplätze auf. An den Universitäten stehen pro Jahr etwa 1050 Medizinstudienplätze zur Verfügung, zur langfristigen Deckung der medizinischen Versorgung der Schweizer Bevölkerung bräuchte es jedoch mindestens 1500. An mangelndem Interesse der Maturanden scheitert es dabei nicht; im vergangenen Jahr gab es mehr als 4000 Bewerber. Doch trotz diverser parlamentarischer Initiativen und Motionen in den letzten Jahren [4] hat sich bis vor kurzem wenig in Richtung der ärztlichen Nachwuchsförderung getan - Gründe dafür sind veranschlagte Kosten von mindestens 300 Millionen Franken, um die Studienplatzkapazitäten zu erhöhen, sowie die Kompetenzverflechtung zwischen Bund und Kantonen.

Der aktuelle Lösungsvorschlag einer vom Bundesamt für Gesundheit und der Schweizerischen Universitätskonferenz geleiteten Arbeitsgruppe kommt nun einem Durchbruch gleich. Statt der Einrichtung zusätzlicher Studienplätze an den bestehenden medizinischen Fakultäten soll das Medizinstudium zukünftig auch ausschliesslich über das Internet absolviert werden können. Die Idee ist nicht neu, sondern greift auf die Erfahrungen mit der jüngsten Studienreform in Finnland zurück (sogenanntes «Finnisches Modell» [5]). Niemand zweifelt mehr ernsthaft daran, dass die erforderlichen Anpassungen des Universitätsförderungsgesetzes in Kürze die parlamentarische Hürde nehmen werden, denn die Kosten für die Einrichtung des virtuellen Studiums sind vergleichsweise niedrig. Um Spannungen zwischen den Universitätskantonen zu vermeiden, sollen fünf medizinischen Fakultämeinsam die Verantworeine befriedigende Ausbiltragen, Praktikumsplätze die Examina abnehmen. ter Anbieter des Internet-baStudiums ist die traditionsund renommierte Fernversität Frauenfeld* [6]. die Gesetzesrevision wie geplant im Frühjahr verabschiedet, können bereits ab dem Sommersemester 


\section{Literatur}

1 Kraft E, Hersperger M. Auch dank Frauen und Ausländern ist die ärztliche Versorgung in der Schweiz garantiert. Schweiz Ärztezeitung; 2011;92(49):1899-901.

2 Kleist P. «Lost in Translation». Babylonische Sprachverwirrung an Schweizer Spitälern. Schweiz Ärztezeitung. 2010;91:143-6.

3 Schweizerisches Institut für Demagogie. Die Entwicklung der Ärztedemographie zwischen 2010 und 2040. Zeitschrift für Medizinische Versorgungsforschung. 2010;5:33A-39A.

4 Motion 08.3608 von Jacqueline Fehr: «Strategie gegen Ärztemangel und Förderung der Hausarztmedizin» (Oktober 2008), Motion 10.3886 der Kommission für Wissenschaft, Bildung und Kultur NR: «Bundeskompetenz für Mindestzahl von Studienplätzen an medizinischen Fakultäten» (Oktober 2010) u.a. www. parlament.ch

5 Hautamäki T, Pesonen J Hämäläinen K. Koirat kutsuen kulkevat kunnon vieraat kutsumatta. Suomen Lääkärilehti. 2008;15:291-8.

6 www.fernuniversität-frauenfeld.ch

7 Getestet an finnischen Studenten, haben sich «Killervirus» (Infektiologie), «SOS Emergency Room» (Notfallmedizin) und «Plagiator II - Fälschern auf der Spur» (Wissenschaftsethik) als didaktisch besonders wertvoll erwiesen.

* Der «Studienleitfaden Medizin» der Fernuniversität Frauenfeld ist als Download unter www. fernuniversitaet-frauenfeld.ch/downloads/ Studienleitfaden/ Leitfaden_Medizin verfügbar.

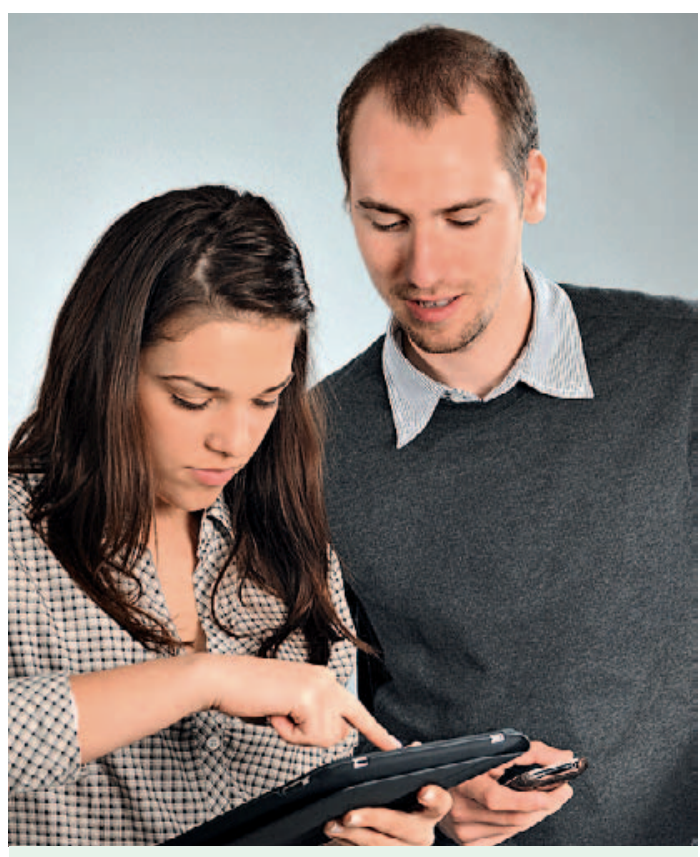

Moderne Technologie mit raffinierten medizinischen Apps macht das Studium von zu Hause aus möglich. Animierte anatomische Modelle und gefilmte chirurgische Eingriffe unterstützen das räumliche Vorstellungsvermögen. Hier zwei Studenten beim Auswerten von Röntgenbildern.

2013 die ersten angehenden Ärztinnen und Ärzte mit dem Studium beginnen. Wegen begrenzter Serverkapazitäten muss die Studentenzahl zunächst auf 250 beschränkt werden.

Voraussichtlich werden den Internet-Studenten der Frauenfelder Fernuniversität zwei unterschiedliche Studiengänge angeboten, die gemäss dem Bolognasystem entweder nach sechs Jahren mit einem Master of Advanced Studies in Human Medicine oder nach drei Jahren mit einem Bachelor in Country Medicine abgeschlossen werden können. Sobald unbegrenzt Studienplätze zur Verfügung stehen, wird für das sechsjährige Studium der Numerus clausus abgeschafft; bis dahin gilt das Losverfahren, d. h. sobald es losgeht, entscheidet das Eingangsdatum der Bewerbung über die Studienplatzvergabe. Die ersten 50 Bewerber sollen zudem ein iPad mit besonderen Apps, zum Beispiel mit Computerspielen aus dem Medizinbereich zur Vorbereitung auf das Studium, erhalten [7]. Für das verkürzte Studium zum «Country Doctor» - hier wird an die bis 1995 geltende, dreijährige Regelstudienzeit für die Humanmedizin angeknüpft - wird zurzeit über die Erfüllung von zwei Zugangsbedingungen diskutiert: Erstens muss der Heimatort der Bewerber in einer Region liegen, in der Ärztemangel herrscht bzw. absehbar ist; zweitens müssen die Studienplatzaspiranten eidesstattlich versichern, sich nach Abschluss ihrer Aus- und Weiterbildung dort einmal niederzulassen. Sollte sich das Konzept in dieser Form durchsetzen, gewährt die Migros-Genossenschaft
Sondernutzungsrechte für ihr Motto «Aus der Region - Für die Region», um das Landarztstudium in der Schweiz bekannt zu machen.

In Frauenfeld sind die Vorbereitungen bereits mit Hochdruck angelaufen. Sobald die gesetzlichen Grundlagen gegeben sind, wird die Fernuniversität an die Öffentlichkeit gehen und für die neuen Studiengänge werben. Dann heisst es: «Mit ein paar Mausklicks zum fertigen Arzt.» Schliesslich ermöglicht das Internet ganz neue Formen des Lernens: animierte anatomische Modelle und gefilmte chirurgische Eingriffe unterstützen das räumliche Vorstellungsvermögen; an virtuellen Patienten erfolgen die Anamneseerhebung und das Abhören von Herztönen; und anhand von Fotos sollen die Studenten lernen, Fusspilz von einer Schuppenflechte zu unterscheiden. Da die neuen Ausbildungsformen hohe EDV-technische Ansprüche an die Studierenden stellen, könnten sie zudem der Feminisierung in der Medizin entgegenwirken - auch wenn «Frauenfeld» etwas ganz anderes vermuten lässt.

An der Fernuniversität ist man aber der Ansicht, dass die Ausbildung, insbesondere beim dreijährigen Bachelor-Studiengang, von unnötigem Ballast zu befreien ist. Die Studierenden sollen sich auf relevante Ausbildungsinhalte konzentrieren, die in der späteren ärztlichen Praxis auch zur Anwendung kommen. So wird es nicht als notwendig erachtet, Strukturformeln auswendig lernen zu lassen; bei Stoffwechselerkrankungen zum Beispiel genügt das Wissen, dass sie irgendetwas mit Chemie zu tun haben. Und zur Diagnose und Behandlung von Bagatellerkrankungen sollte das vorauszusetzende, durchschnittliche Allgemeinwissen ausreichen. Allerdings ist es im Hinblick auf die zukünftige Tätigkeit notwendig, den Blick auch über den Tellerrand hinaus zu richten. Für die Ausbildung in Country Medicine macht es beispielsweise durchaus Sinn, per Video dem Tierarzt-Kollegen bei der Geburt eines Kalbes über die Schulter zu schauen, um später eine umfassende medizinische Versorgung zu gewährleisten.

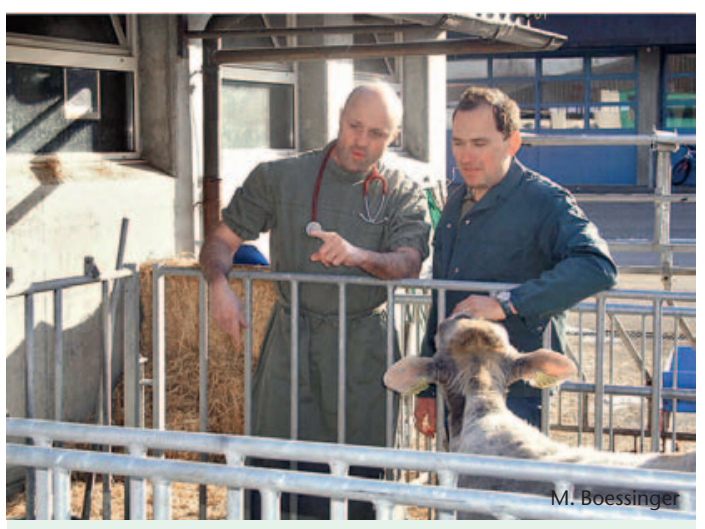

Nach dem Hausbesuch bei der Grossmutter wirft der Doktor noch einen Blick in den Stall. Das Internet-Studium zum Bachelor in Country Medicine bereitet zukünftige Ärzte besser auf diese Situation vor. 